\title{
VRÁBLE-FIDVÁR IM KONTEXT: ZU VORLÄUFERN UND PARALLELEN DER DEMARKATION
}

\author{
KLÁRA P.FISCHL - GIA N-LUCA PAUL - TOBIAS L. KIEN L I N
}

\begin{abstract}
Vráble-Fidvár in Context: Forerunners and Parallels of Its Demarcation. In this contribution in honour of our esteemed colleague and friend Jozef Bátora we take Vráble-Fidvár, one of his most widely known field-projects and excavations, as a starting point for a short review and comparison of the demarcations surrounding and structuring the tell or tell-like multi-layer settlement mounds and their outer settlement of the various Early Bronze Age groups of the Carpathian Basin. From a mainly Hungarian perspective, and with a special focus on the recent results of the BORBAS project (Borsod Region Bronze Age Settlement), attention is drawn to both structural similarities and variability in the way these communities enclosed their sites or a part thereof, and how these settlements developed through time. Furthermore, attention is also drawn to the group of preceding Neolithic enclosures that likewise surround multi-layer settlement mounds. These are distant forerunners of the phenomenon of Bronze Age demarcations studied, but they nonetheless often expose structural similarity and were also subject to change through time and modification in consequence of the permanent negotiation of social space in their respective communities.
\end{abstract}

Keywords: Hungary, Borsod Plain, Bronze Age, tell settlements, demarcation.

\section{EINLEITUNG}

Auf die Ergebnisse der ersten Grabungen in Vráble-Fidvár von $A$. Točík (1968) folgten in den letzten Jahren bemerkenswerte Publikationen, die Luftbildaufnahmen sowie geophysikalische Prospektionen umfassten und das dreigliedrige Grabensystem des Fundortes aufzeigten (Bátora u. $a$. 2008; 2012; Bátora/Tóth/Rassmann 2015; Falkenstein u.a. 2008; Rassmann u. a. 2017; 2018; Schlüt/Bittmann 2016; Skorna/Kalmbach/Bátora 2018). Den neueren Untersuchungen zufolge ließen sich den drei den inneren Siedlungskern umgebenden Gräben verschiedene Zeitstellungen (BA1-BB1) und Kulturgruppen zuordnen (Abb. 1). Während die früheste nachgewiesene Siedlungsaktivität in Vráble-Fidvár in den Makó-Kosihy-Čaka-Horizont datiert werden kann, wurde der innerste und zugleich älteste Graben A samt eines Erdwalls zu Beginn der Frühbronzezeit von Trägern der Hatvan-Kultur angelegt. Der äußerste Graben C umschloss während der folgenden zweiten Siedlungsphase, die der Aunjetitzer-Kultur zugeschrieben werden konnte, die größte Ausdehnung der Siedlung. Der innere Graben A wurde zur selben Zeit mit Siedlungsmaterial beider Kulturgruppen verfüllt. In der dritten und letzten Siedlungsphase kam es zur Reduktion des besiedelten Areals und dem damit einhergehenden Aushub des massiven Grabens B. Aufgefundene Keramik der Madarovce-Kultur ermöglichte eine Datierung an das Ende der Frühbronzezeit. Vermutlich erst nach Aufgabe der Siedlung kam es zur allmählichen Verfüllung von Graben B durch natürliche Erosionsprozesse.
Neben dem sich dynamisch entwickelnden Grabensystem rücken zwei weitere charakteristische Elemente in den Fokus der Untersuchungen. Zum einen scheinen einige Häuser radial um den Graben angeordnet gewesen zu sein. Inwieweit ein solcher systematischer Aufbau bereits zur hatvanzeitlichen Besiedlung bestand, ist aufgrund der durch Erosion zerstörten Kulturschichten des zentralen Siedlungsareals nicht $\mathrm{zu}$ bestimmen. Zum anderen fand sich jenseits der Gräben in der Außensiedlung ein offensichtlich separierter Bereich, in dem zahlreiche Grubenbefunde nachgewiesen werden konnten.

Die Siedlung Vráble-Fidvár befindet sich im Südwesten der Slowakischen Ebene oberhalb der rezenten Flussaue der Žitava und in unmittelbarer Nähe zu den polymetallischen Lagerstätten der angrenzenden Westkarpaten, die Vorkommen von Gold- und Zinnseifen sowie sulfidischen Kupfererzen umfassen (Bátora u. a. 2008, Abb. 1). Die fruchtbaren Lössböden der umgebenden Terrassenlandschaft boten darüber hinaus eine geeignete Subsistenzgrundlage, wie schon die kontinuierliche Nutzung des Umfeldes von Vráble-Fidvár durch verschiedene Kulturgruppen nahelegt. Für die günstigen Siedlungsbedingungen dieser Mikroregion sprechen so etwa eine neolithische Siedlung der Linienbandkeramischen Kultur ebenso wie ein römisches Militärlager, die unweit von Vráble-Fidvár bestanden und im Rahmen der geomagnetischen Prospektion nachgewiesen wurden (Furholt u. a. 2014). Bemerkenswert ist ferner, dass die Siedlung einen Kontaktpunkt der aus dem Theiß-Gebiet stammenden Hatvan-Kultur und der 


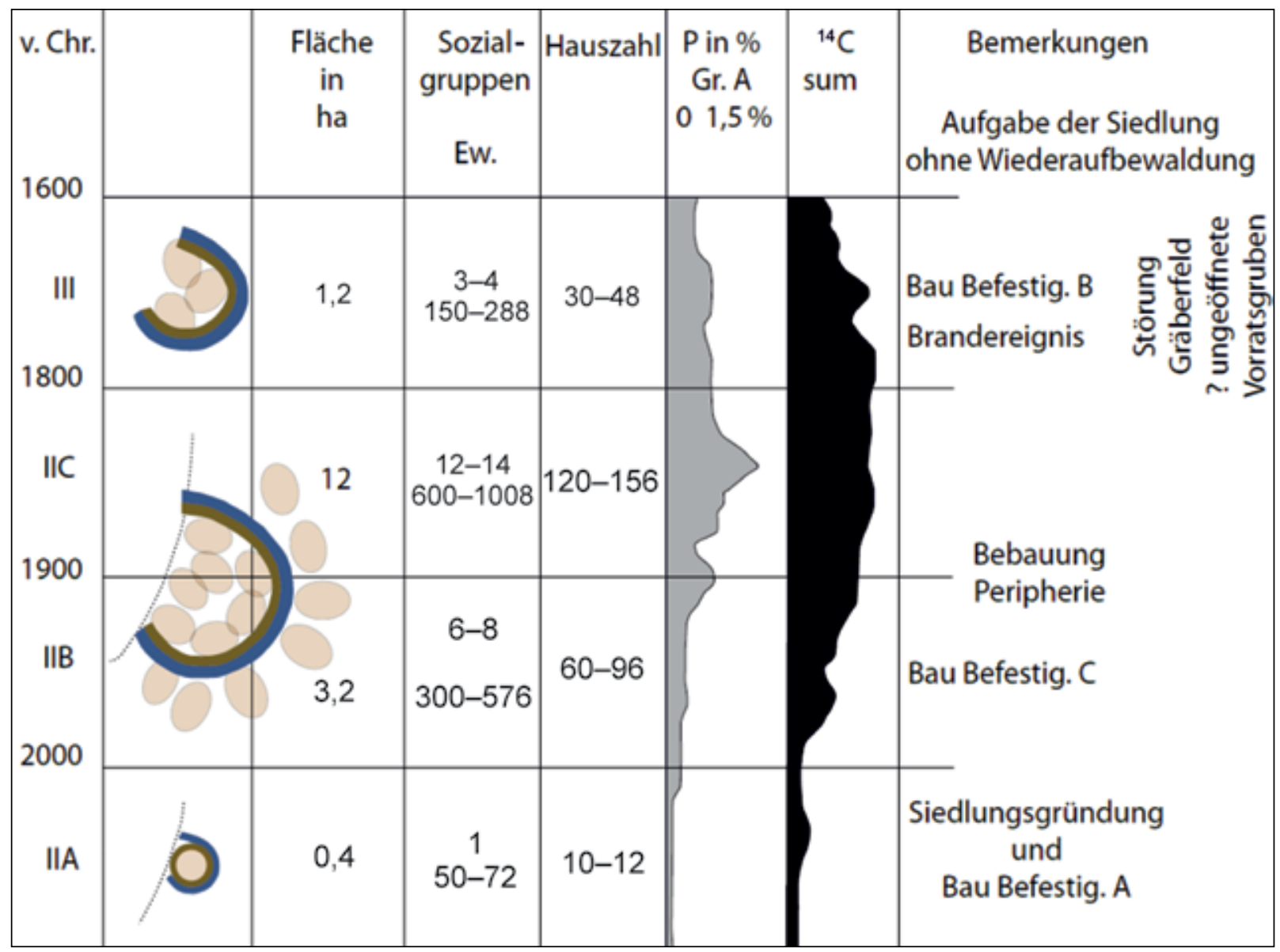

Abb. 1. Phasen und Ausdehnung der Siedlung Vrable-Fidvár (nach Skorna/Kalmbach/Bátora 2018, 103).

mitteleuropäischen Aunjetitzer-Kultur darstellt und das jeweils äußere Verbreitungsgebiet der beiden Kulturgruppen markiert.

\section{PARALLELEN}

Das dynamische Grabensystem Vráble-Fidvárs findet im südöstlichen Mitteleuropa nur wenige Parallelen. Eine Ausnahme bildet die Siedlung Včelince-Lászlófala, die eine vergleichbare, mehrphasige Grabenstruktur sowie eine Nutzung durch verschiedene Kulturgruppen aufwies (Furmánek/ Marková 1992; 1998; 2001; 2008; Görsdorf/Markovál Furmánek 2004). Die drei Gräben ließen sich der hatvanzeitlichen Besiedlung während der Frühbronzezeit zuordnen. Trotz der Parallelen zu Vráble-Fidvár erlaubt der momentane Publikationsstand von Včelince-Lászlófala keine weiteren Vergleiche zwischen diesen beiden Siedlungen.

N. Kalicz stellte bereits 1968 heraus, dass für nahezu alle Siedlungen der Hatvan-Kultur Grabenanlagen charakteristisch seien, die den zen- tralen Siedlungskern, nicht jedoch die umgebende Außensiedlung einfassen würden (Kalicz 1968, 132-134). Auf Grundlage dieser Beobachtungen wurde 2012 in Kooperation zwischen der Universität zu Köln, der Universität Miskolc sowie dem Herman Ottó Museum in Miskolc das „Borsod Region Bronze Age Settlement"-Projekt (BORBAS) gegründet, dessen vorrangiges Ziel in der Rekonstruktion des früh- und mittelbronzezeitlichen Siedlungswesens zwischen der Fußhügelzone des Bükk-Gebirges und der südlichen Borsodebene liegt (Fischl/Kienlin/Pusztai 2016; Kienlin/Fischl/ Pusztai 2018). Innerhalb dieser Mikroregion im Nordosten Ungarns konnten bislang 17 Fundorte der Hatvan- und Füzesabony-Kultur identifiziert und insbesondere durch geomagnetische Prospektion untersucht werden (Abb. 2). Seit 2018 finden zudem systematische Kernbohrungen statt, die primär die Ausmaße der Gräben sowie die absolute Datierung und das chronologische Verhältnis von zentralem Tell, Demarkation und umgebender Außensiedlung innerhalb des Siedlungsgefüges erfassen sollen. 


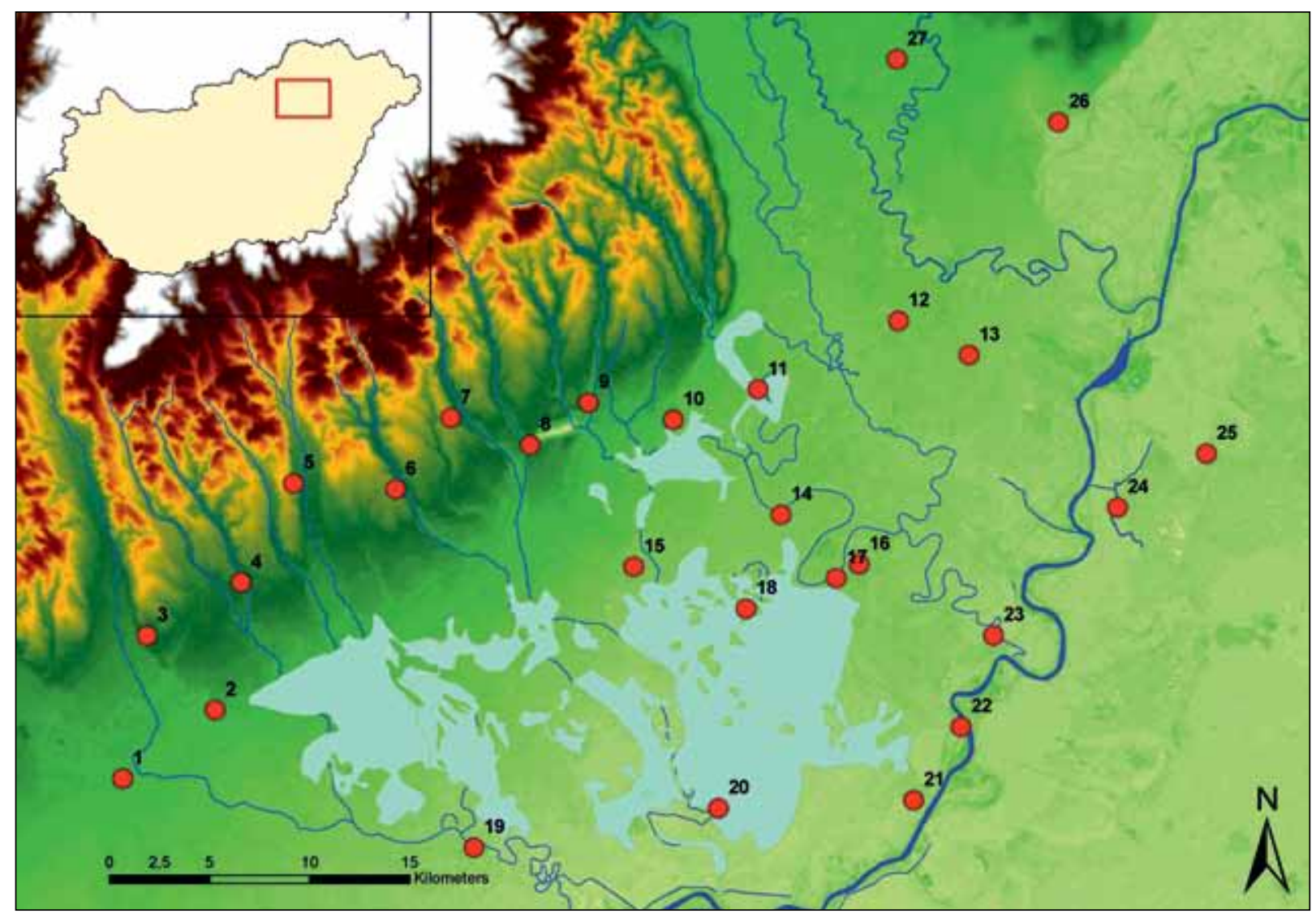

Abb. 2. Verbreitungskarte der bekannten hatvan- und füzesabonyzeitlichen Siedlungen auf der Borsodebene. 1 - Füzesabony-Öregdomb; 2 - Szihalom-Földvár; 3 - Maklár-Földvár; 4-Novaj-Földvár; 5 -Bogács-Pazsagpuszta; 6 - Tard-Tatárdomb; 7 - Tibolddaróc-Bércút; 8 - Bükkábrány-Kálvária; 9 - Vatta-Testhalom; 10 - Emőd-Nagyhalom; 11 - Emőd-Karola szőlők; 12 - Szakáld-Testhalom; 13 - Sajószöged-Aranyos halom; 14-Mezőcsát-Laposhalom; 15 -Gelej-Pincehát; 16 - Mezőcsát-Oroszdomb; 17 - Mezőcsát-Pástidomb; 18 - Mezőcsát-Harangdomb; 19 - Borsodivánka-Marhajárás (Nagyhalom); 20 - Tiszabábolna-Fehérló tanya; 21 - Ároktő-Dongóhalom; 22 - Tiszakeszi-Szódadomb; 23 - Tiszakeszi-Bálinthát (Újtemető); 24 - Polgár-Ásott halom; 25 - Polgár-Kenderföld; 26 - Tiszalúc-Dankadomb; 27 - Hernádnémeti-Németi halom.

Die zentralen Siedlungshügel der prospektierten Fundorte wiesen Flächen von durchschnittlich 0,5 ha auf (vgl. Kienlin/Fischl/Pusztai 2018, 56, 83, Tab. I-4; Abb. I-51) und waren von einem zwischen $10 \mathrm{~m}$ und $20 \mathrm{~m}$ breiten Graben eingefasst. In einigen der umgebenden Außensiedlungen, die den größten Teil der Siedlungsfläche ausmachten, konnten Nachweise von (verbrannten) Hausbefunden erbracht werden. Sie waren rund 4-5 m breit, 10-16 m lang und meist nord-südlich beziehungsweise nordwest-südöstlich ausgerichtet.

Im Kontrast zu dieser verhältnismäßig streng befolgten, normativen Grundstruktur findet sich in der Borsodebene sowohl zwischen den Fundorten als auch in der Entwicklung einzelner Siedlungen ein höheres Maß an Variabilität als bisher angenommen. Durchaus vergleichbar zu Vráble-Fidvár waren auch die Hausstrukturen im inneren Bereich der zonierten Außensiedlung von Emőd-Nagyha- lom sowie in der Außensiedlung von Tard-Tatárdomb konzentrisch um den umgebenden Graben angeordnet (Abb. 3). Dabei waren sie jedoch konträr zu Vráble-Fidvár jeweils mit ihren Längsseiten zum Graben hin ausgerichtet. In Vatta-Testhalom dagegen behielten die Häuser ihre grundsätzliche, nordwest-südöstliche Ausrichtung bei (Abb. 3). Im äußeren Bereich der Außensiedlungen von Emőd-Nagyhalom und zum Beispiel in Tiszakeszi-Bálinthát-Újtemető standen die Häuser parallel zueinander in Reihen, während sie in Tibolddaróc-Bércút einzelne und voneinander separierte Gruppen bildeten (Kienlin/Fischl/Pusztai 2018).

Wohl alle der bislang untersuchten Siedlungen der Borsodebene wiesen eine kontinuierliche Nutzung durch die Hatvan- und die jüngere Füzesabony-Kultur auf, und in einigen von ihnen konnten Modifikationen an den Grabenanlagen nachgewiesen werden. Zu dieser Gruppe gehört 


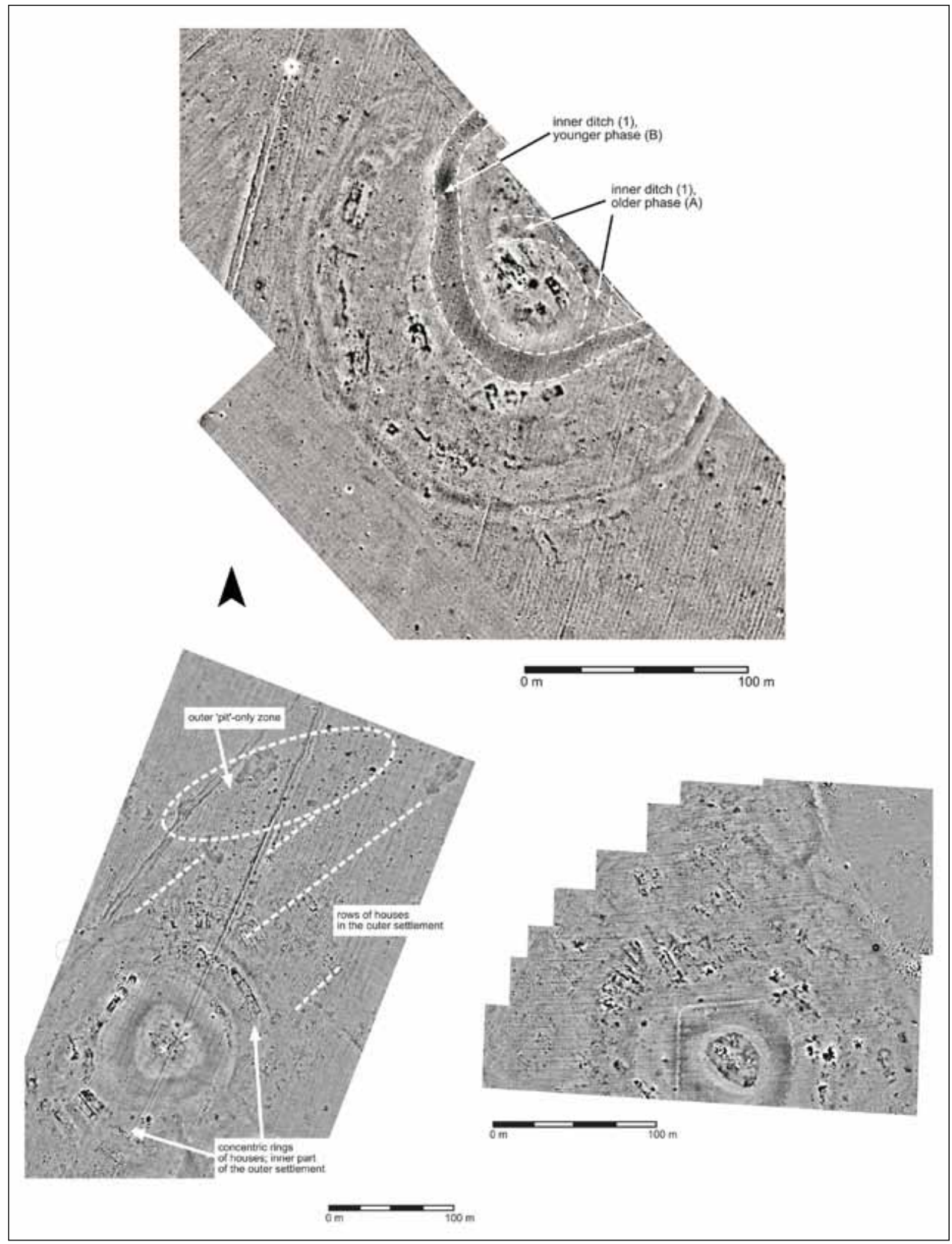

Abb. 3. Magnetogramme der bronzezeitlichen tellartigen Siedlungen von Tard-Tatárdomb (oben), Emőd-Nagyhalom (unten links) und Vatta-Testhalom (unten rechts) einschließlich der umlaufenden Demarkationen sowie der Außensiedlungen. Quelle: BORBAS project. 
neben Ároktő-Dongóhalom und Szakáld-Testhalom sicher auch Tard-Tatárdomb, dessen hatvanzeitlicher Siedlungskern zunächst von einem rundlichen Graben umgeben war. Kernbohrungen, die im Jahr 2019 durchgeführt wurden, belegten anschließende Modifikationen am Graben, bei denen der nördliche und vermutlich auch der östliche Bereich der Demarkation teilweise verfüllt und im Norden nach außen hin vergrößert wurde (siehe auch bereits Fischl/Kienlin/Seres 2012; Kienlin/Fischl/Pusztai 2018). Von der nachfolgenden Besiedlung zeugen neben den zumeist wohl umgelagerten Kulturschichten im Bereich des verfüllten Grabenteils insbesondere die in die Verfüllungsschicht eingetieften und im Magnetogramm erkennbaren Gruben.

Eine andere Situation, die bisher im Forschungsgebiet ohne Parallelen ist, ergab sich in der Siedlung Emőd-Nagyhalom. Der zentrale, tellartige Siedlungshügel besaß eine Fläche von rund 0,26 ha und war von einem 14-20 m breiten Graben eingefasst, der sowohl im Magnetogramm als auch im Gelände selbst gut sichtbar ist (Abb. 3). Erst anhand der systematischen Kernbohrungen im Jahr 2018 konnte nachgewiesen werden, dass der Graben in einer älteren Siedlungsphase einen weitaus breiteren Vorläufer besaß, der um die Mitte des 20. Jhs. v. Chr. von außen partiell verfüllt wurde. Auf dieser Verfüllung entstanden wohl mit einigem zeitlichen Abstand während der zweiten Hälfte des 19. Jhs. v. Chr. Häuser, die sich, wie bereits geschildert, konzentrisch um den Graben orientierten (Abb. 3; Kienlin/Fischl/Pusztai 2018; Kienlin/Lie/Fischl 2019). Solche Modifikationen der Gräben entsprechen offenkundig nicht derselben Dynamik, wie sie in Vráble-Fidvár belegt ist. Vergleichbar ist neben der grundsätzlichen Siedlungsstruktur hingegen, dass auch auf den Fundorten der Borsodebene gelegentlich eine separierte "Grubenzone" in der Außensiedlung nachgewiesen werden kann (Fischl/ Kienlin 2013, 8).

Entsprechende Grabensysteme sind allerdings nicht nur auf der Borsodebene oder innerhalb des Hatvaner Kulturkreises kennzeichnendes Element der Tell- und tellartigen Siedlungen, sondern sie finden sich auch in angrenzenden Regionen des Karpatenraums wieder. Die vermehrte Nutzung nicht-invasiver Maßnahmen, wie Luftbildaufnahmen oder geophysikalische Prospektionen, lieferte in den letzten Jahren immer mehr einschlägige Daten, die zu einem besseren Verständnis dieser Anlagen beitragen. Ein- oder mehrfache Grabensysteme der Aunjetitzer-Kultur konnten beispielsweise jüngst in Tschechien identifiziert werden (Kovárník 2015). Ähnliches gilt für die Siedlung Budmerice der Madarovce-Kultur, die ein komplexes System aus insgesamt vier Gräben aufweist (Vavák u. a. 2015).
Eine weitere Besonderheit, die sich in einigen Siedlungen der Vatya-Kultur an der mittleren Donau findet, stellen Grabensysteme dar, die die interne Siedlungsfläche in mehrere, womöglich funktional unterschiedlich genutzte Zonen untergliederten (Jaeger u. a. 2018; Staniuk u. a. 2020; Szeverényi/Kulcsár 2012). Auch in der als Polgár-Insel bekannten Mikroregion östlich der Theiß konnten in den letzten Jahren vermehrt Siedlungen identifiziert und untersucht werden, die massive und teils mehrere Gräben aufwiesen. Vergleichbar zu den Fundorten der Borsodebene bestanden einige dieser Ansiedlungen kontinuierlich während der Hatvan- und Füzesabony-Phase (Dani u. a. 2019). Grabensysteme um mehrschichtige Ansiedlungen des Otomani-Füzesabony-Kulturkomplexes finden sich zudem an den Flussläufen der Berettyó (Dani 2012) und des Ér/ Ier (Gogâltan/Cordoș/Ignat 2014; Kienlin/Fischl/Marta 2017) im Osten der Ungarischen Tiefebene sowie in weiten Teilen der heutigen Slowakei. Dort wies die Siedlung Nižná Myšla einen inneren Siedlungskern von rund 0,3 ha Fläche auf, der zunächst von einem massiven Graben umfasst war. Vergleichbar zu Vráble-Fidvár kam es in einer jüngeren Siedlungsphase zu einer deutlichen Ausdehnung des besiedelten Areals, infolge derer ein neuer, rund

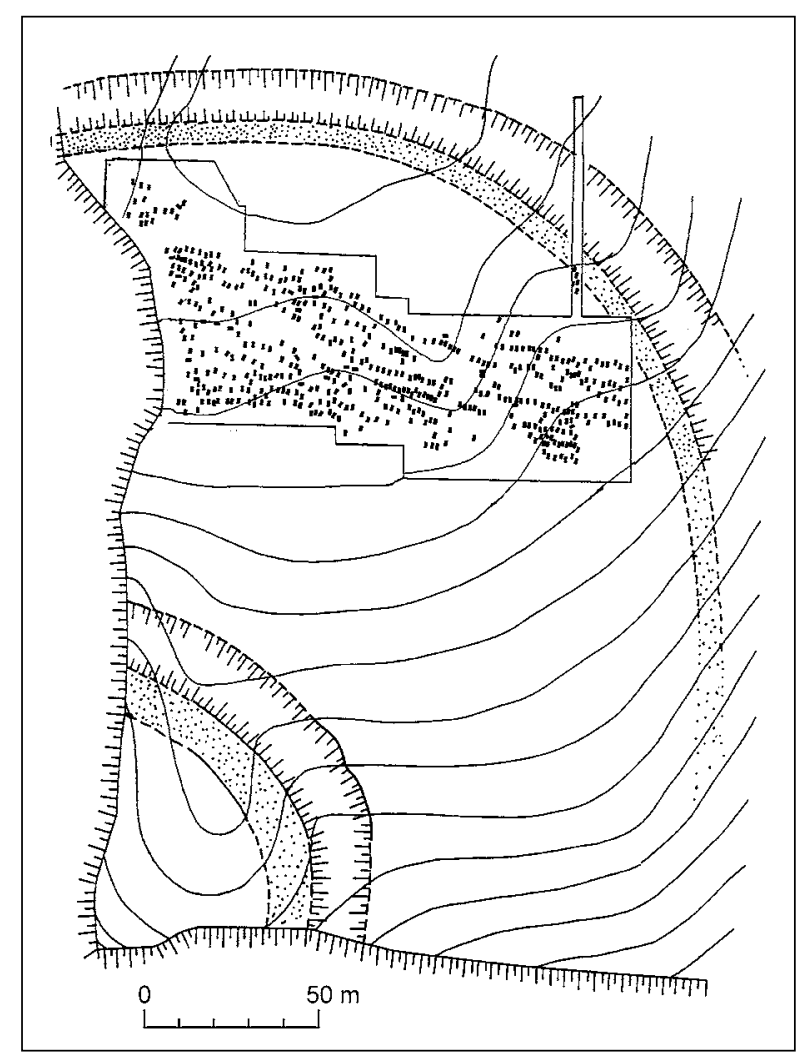

Abb. 4. Die zweiphasige Grabenanlage von Nižná Myšl’a (modifiziert nach David 2010, Abb. 4). 

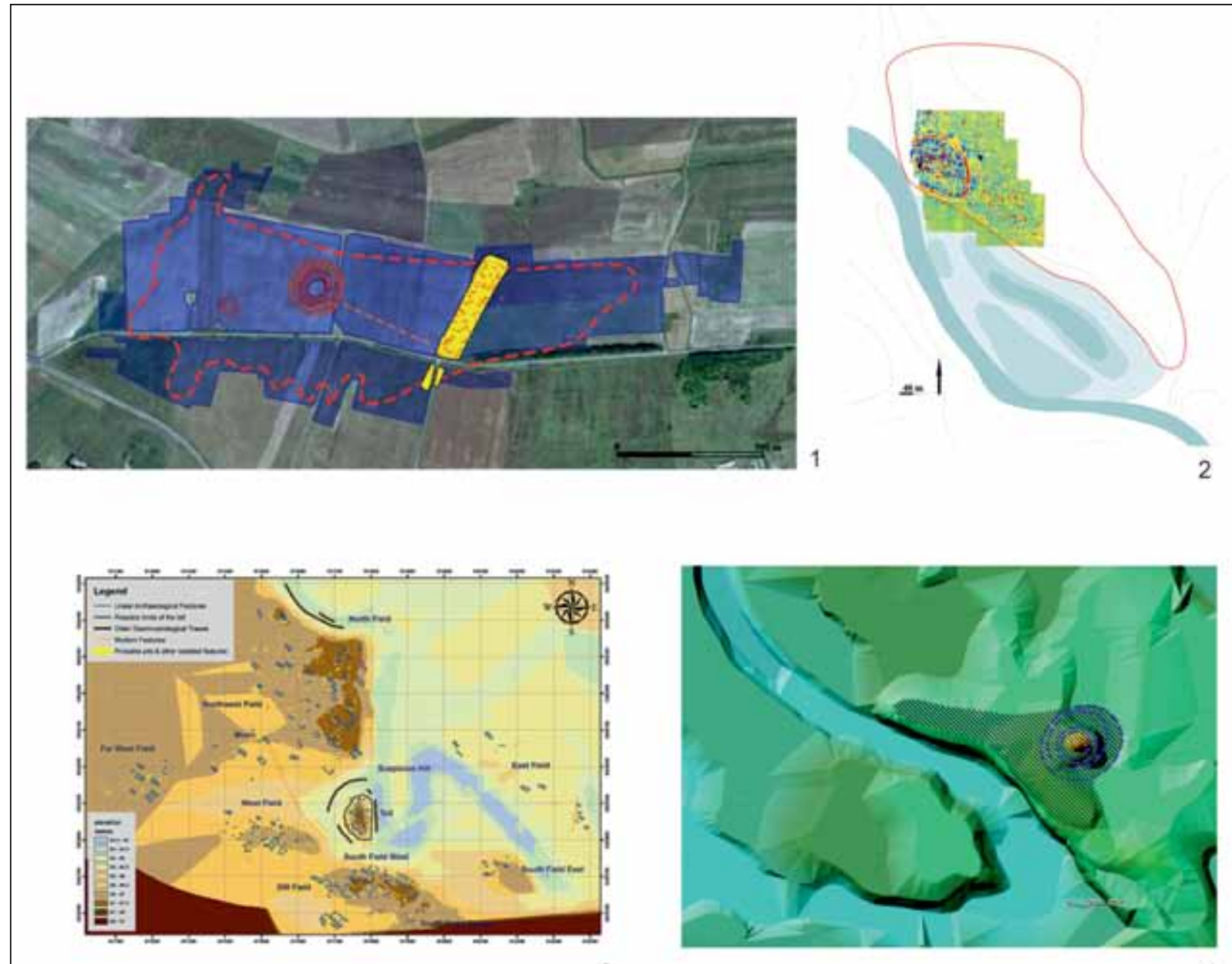

3
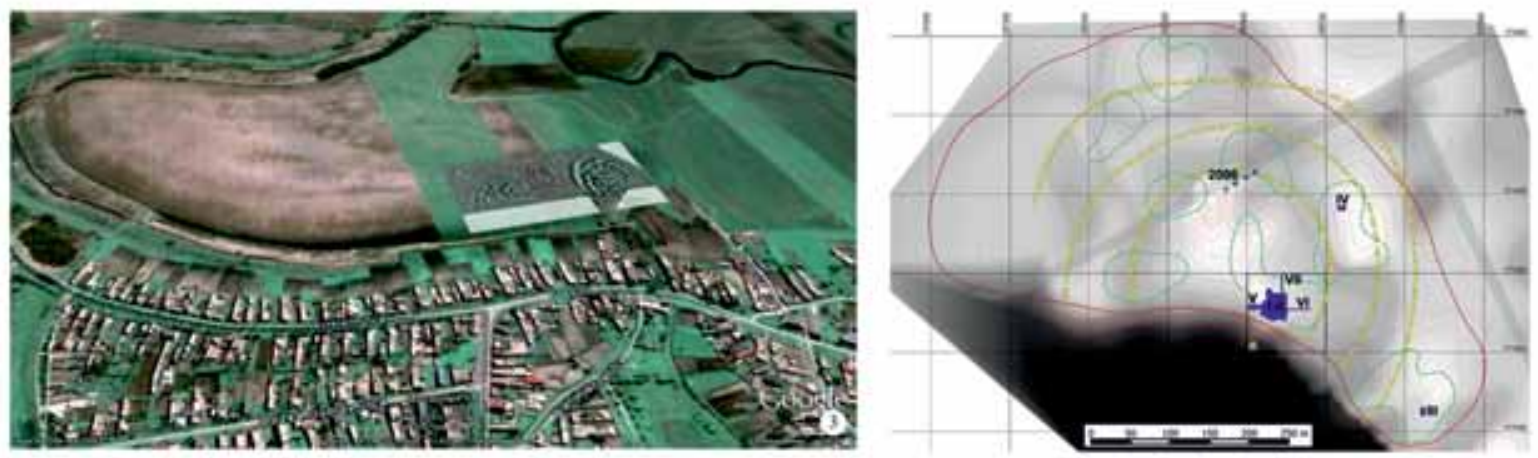

5

Abb. 5. Demarkationen der neolithischen Tell- und tellartigen Siedlungen. 1 - Polgár-Csőszhalom (nach Mesterházi u. a. 2019, Abb. 7); 2 - Polgár-Bosnyákdomb (nach Raczky/Anders 2009, Abb. 4); 3 - Szeghalom-Kovácshalom (nach Gyucha u. a. 2015, Abb. 4); 4 - Hajdúböszörmény-Pródi-halom (nach Raczky/Fodor/Mester 2010, Abb. 12); 5 - Szentpéterszeg-Kovadomb (nach Raczky/Anders 2014, Abb. 3); 6 - Öcsöd-Kováshalom (nach Füzesi/Raczky 2018, Abb. 1). 
$27 \mathrm{~m}$ breiter und $5 \mathrm{~m}$ tiefer Graben am Rande der Siedlung ausgehoben wurde (Abb. 4; Fischl/Olexa 2019; Gašaj 2002; Olexa 1982; 2003).

Der Siedlungstyp des kontinuierlich belegten, mehrschichtigen Siedlungshügels einschließlich der zugehörigen Demarkationen scheint somit während der frühen bis mittleren Bronzezeit (ungarischer Terminologie) regional wie auch chronologisch innerhalb des Karpatenraums Variation unterworfen zu sein, wenngleich bestimmte strukturelle Merkmale gleichsam in den meisten Siedlungen zu beobachten sind.

\section{VORLÄUFER}

Grabenanlagen sind innerhalb des Karpatenraums bereits aus dem vorangehenden Neolithikum bekannt. So weisen schon zahlreiche Siedlungen der Linienbandkeramischen Kultur eine äußere Demarkation auf (Raczky/Anders 2012). Insbesondere für das Spätneolithikum steht dann eine stetig zunehmende Menge an Daten zur Verfügung, die ein besseres Verständnis des Siedlungswesens ermöglicht. Die Region entlang der Theiß im Osten des heutigen Ungarns bildet exemplarisch für diesen Zeitraum eine archäologisch gut fassbare, jedoch uneinheitliche Siedlungslandschaft. Telloder tellartige Siedlungen finden sich hier nahezu ausschließlich im mittleren und südlichen Abschnitt der Theiß sowie entlang der Körös-Flussläufe, während im Norden Flachsiedlungen zu dominieren scheinen (Raczky 1987). Des Weiteren unterscheiden sich die Tell- und tellartigen Ansiedlungen der Berettyó-Gegend von denen der südlich gelegenen Tiefebene (Kalicz 1985; Kalicz/Raczky 1990). Vergleichbar zur Bronzezeit scheint somit auch im spätneolithischen Siedlungswesen und innerhalb einzelner Mikroregionen Variabilität bestanden zu haben.

Dem allgemein geläufigen Modell zufolge besitzen diese Tellsiedlungen gesellschaftliche, ökonomische sowie soziale Funktionen und sind von sogenannten Satellitensiedlungen umgeben. Neuere Untersuchungen - beginnend mit denen der Polgár-Mikroregion - deuten darauf hin, dass die Tellsiedlungen als Zentren großflächiger, horizontaler Siedlungsräume umrissen werden können und von diesen durch Grabenanlagen separiert waren. Neben dem für die sogenannte Csőszhalom-Gruppe eponymen Fundort Polgár-Csőszhalom am Oberlauf der Theiß weisen auch Polgár-Bosnyákdomb, Szentpéterszeg-Kovadomb, Hajdúböszörmény-Pródi-halom und Szeghalom-Kováshalom (Gyucha u.a. 2015; Raczky/Anders 2009; 2014; Raczky/Fodor/Mester 2010) sowie weitere, unpublizierte Siedlungen eben- jene Struktur auf (Abb. 5). Eine andere Raumorganisation findet sich hingegen beispielhaft in Vésztö-Mágor (Gyucha u. a. 2015) oder Öcsöd-Kováshalom. Der tellartige Siedlungshügel von Öcsöd-Kováshalom war dabei von drei konzentrisch verlaufenden, jedoch segmentierten Gräben sowie von sieben weiteren, zeitgleich bestehenden Siedlungs- bzw. Haus-Nuclei umgeben. Die Schichtmächtigkeit des zentralen Siedlungshügels betrug $130-160 \mathrm{~cm}$, die Gesamtfläche des genutzten Areals rund 21 ha (Füzesi/Raczky 2018; Raczky 1987; Raczky/Füzesi 2016; Raczky/Füzesi/Anders 2018).

In Polgár-Csőszhalom umschlossen den tellartigen Siedlungshügel insgesamt sechs konzentrische Gräben, die während verschiedener Siedlungsphasen bestanden und das Ergebnis einer episodischen Ausdehnung des zentralen, kultisch genutzten Areals waren (Raczky/Anders 2010a). Erste systematische Ausgrabungen fanden 1957 unter der Leitung von I. Bognár-Kutzián statt (Bánffy/Bognár-Kutzián 2007). Umfangreiche Informationen zur Tell- und umgebenden Außensiedlung erbrachten weitere Maßnahmen, die seit 1989 und insbesondere infolge des geplanten Baus der M3-Autobahn fortgeführt wurden. Das gesamte Siedlungsareal umfasste den neueren geomagnetischen Untersuchungen zufolge rund 67,5 ha (Mesterházy u. a. 2019). Die Häuser, die innerhalb der Grabenanlage bestanden, waren radial zu ihnen angeordnet, während diese in der Außensiedlung einer nordwest-südöstlichen Ausrichtung folgten. Nach Meinung der Ausgräber soll sich die Gesamtsiedlung von Polgár-Csőszhalom durch eine ausgesprochene Dualität bezüglich der Raumkonzeption auszeichnen (Raczky/Anders 2010b; 2012; Raczky/Sebő́k 2014). Während der mehrschichtige Siedlungshügel dem Aufbau der Tell-und tellartigen Siedlungen der Theiß-Kultur an der mittleren und unteren Tisza entspräche, würden die mehrgliedrigen Grabenanlagen vielmehr Parallelen in den sogenannten Rondellen der Lengyel-Kultur finden (Barna u. a. 2016; Pásztor/Barna/Zotti 2015), sodass Polgár-Csőszhalom als kultureller Verbindungspunkt zwischen dem westlichen Transdanubien und der östlichen Tisza-Gegend anzusehen sei (Raczky/Anders 2006; 2008; Raczky/Anders/Bartosiewicz 2011). Die geographische Lage, kultbezogene Funde und Befunde sowie die Raumkonzeption führten über einen längeren Zeitraum dazu, Polgár-Csőszhalom eine Sonderstellung innerhalb der bekannten, spätneolithischen Siedlungslandschaft zukommen zu lassen (Mesterházy u. a. 2019; Raczky 2019; Raczky/Anders 2010b; 2012; Raczky/Sebők 2014; Raczky/Anders/Siklósi 2014; Raczky u. a. 2015). Die beispielhaft vorgestellten Fundstellen ergeben jedoch ein differenzierteres Bild, das auf allgemeine Raumkonzeptionen und Denkweisen neolithischer 
Gesellschaften ebenso verweist wie auf Spezifika einzelner Anlagen oder Mikroregionen.

Trotz des zwischenzeitigen Verschwindens der Tellsiedlungen am Ende des Spätneolithikums finden sich auch während der nachfolgenden Kupferzeit innerhalb des Karpatenraums weiterhin Siedlungen, die von Gräben umgeben waren (Parkinson u. a. 2004).

\section{DEUTUNG}

Vráble-Fidvár wird aufgrund der sprunghaften und sich dynamisch entwickelnden Siedlungsausdehnung sowie des normiert wirkenden Aufbaus eine hervorgehobene Bedeutung als frühbronzezeitliches Siedlungszentrum zuerkannt. Die im Rahmen dieses Beitrags gegenübergestellten Siedlungsformen unterschiedlicher Kulturgruppen, Regionen und Zeitstellungen lassen einerseits in der Entwicklung einzelner Siedlungen sowie zwischen verschiedenen Fundorten ein hohes Maß an Variabilität ersichtlich werden. Anderseits weisen viele mit Gräben umgebende bronzezeitliche Tellsiedlungen des Karpatenraums - und unter ihnen auch Vráble-Fidvár - bestimmte strukturelle Merkmale gemeinsam auf, die Parallelen bereits im vorangehenden Neolithikum haben. In Vráble-Fidvár gehört zu diesen insbesondere die systematische Anordnung der Häuser entlang des Grabens und innerhalb der umgebenden Außensiedlung, die, ebenso wie die Größe des zentralen, hatvanzeitlichen Siedlungshügels, Entsprechungen in den hatvanzeitlichen Siedlungen der Borsodebene finden. Eine zu Vráble-Fidvár vergleichbare sprunghafte Siedlungsausdehnung ist hingegen auf der Borsodebene nicht festzustellen, sie ist jedoch beispielsweise in der Otomani-Füzesabony Siedlung von Nižná Myšla zu beobachten.

Die meisten der Tell- oder tellartigen Siedlungen waren - wie auch Vráble-Fidvár - von einer Grabenanlage umgeben. Diese sind jedoch nicht allein für die Bronzezeit, sondern ebenso für die spätneolithischen, mehrschichtigen Ansiedlungen charakteristisches Merkmal. Die Anzahl und Größe der jeweiligen Gräben variierte - einige bestanden während der gesamten Laufzeit der zugehörigen Siedlung, andere wiederum wurden zu einem späteren Zeitpunkt angelegt oder nachträglich verändert. Forschungsgeschichtlich betrachtet werden Gräben oder Wall-Grabensysteme oft als reine Defensivund Zwangsarchitektur gedeutet, die dem Schutz des umgebenden Territoriums und der Kontrolle der umgebenden Außensiedlung gedient habe. Die Errichtung solcher Befestigungen müsse zentral von einer elitären Schicht organisiert worden sein, sodass befestigte Siedlungen als Zeichen sozialer Hierarchisierung gewertet werden könnten.

Demarkationen, wie sie die Gräben der prähistorischen Siedlungen darstellen, begrenzten und untergliederten die nutzbare Siedlungsfläche und prägten auf diese Weise maßgeblich das entstehende Siedlungsbild. Dieses unterlag jedoch nicht zwingend einer urbanistischen und von "oben" gelenkten Konzeption, sondern mag ebenso gut geteilte Traditionen oder gemeinschaftliche Anliegen zum Ausdruck bringen, die sozial oder auch funktional begründet sein konnten. Spezifische Praktiken, bestimmte Denkweisen oder Raumvorstellungen einer Gemeinschaft schlugen sich in der jeweiligen Siedlungsstruktur nieder. So können Modifikationen an Gräben, wie sie unter anderem in der Borsodebene nachgewiesen und weiter oben exemplarisch dargestellt wurden, auf geänderte Lebensumstände, Bedürfnisse oder Vorstellungen der Bewohner der Siedlung zurückzuführen sein, mit denen sich ebenso die ursprüngliche Gestaltung oder gar Funktion sowie die Wahrnehmung und Bedeutung der Demarkation gewandelt haben mögen. Aus diesen Gründen ist eine Beurteilung der Gräben als rein fortifikatorisches Architekturelement als zu statisch und einseitig anzusehen.

In zahlreichen von unterschiedlichen (ursprünglich keramisch definierten) Kulturgruppen nachfolgend genutzten Siedlungen besteht das ursprüngliche Siedlungsbild zumindest in Grundzügen fort. Solche streng befolgten Siedlungsstrukturen innerhalb gut abgrenzbarer Mikroregionen ebenso wie überregionale Entsprechungen zwischen verschiedenen Kulturgruppen verweisen auf starke Kommunikationsnetze sowie auf gemeinsame Denkmuster und eine geteilte Auffassung von der umgebenden Umwelt. Der geographische und kulturelle Kontext trägt daher zu einem besseren Verständnis auch der Siedlung Vráble-Fidvár bei. Ihre Besonderheit liegt vornehmlich in der Lage zwischen zwei unterschiedlichen Kulturräumen. Ein vergleichbares Bild ergibt sich entlang des Hrons in einem östlich der Žitava gelegenen Tal, in dem die Madarovce-Kultur und die transdanubische Kultur der Inkrustierten Keramik nebeneinander siedelten, was die vorteilhaften naturräumlichen Begebenheiten dieser Mikroregion zudem hervorhebt.

Von diesem ausgehend ist es möglich den Fundort Vráble-Fidvár als einen wichtigen Verbindungspunkt von östlichen und westlichen Kulturräumen zu identifizieren, der sich im verhältnismäßig streng befolgten strukturellen Umfeld der bronzezeitlichen Tell-Siedlungen des Karpatenraums wiederfindet. 


\section{LITERATUR}

Bánffy/Bognár-Kutzián 2007 - E. Bánffy/I. Bognár-Kutzián: The Late Neolithic Tell Settlement at Polgár-Csöszhalom. The 1957 Excavation. BAR 1730. Oxford 2007.

Barna u. a. 2016 - J. P. Barna/Z. M. Tokai/E. Pásztor/I. Eke/ S. Puszta/A. Puszta/J. Busznyák/K. T. Biró/C. Száraz: Late Neolithic Circular Ditch Systems in Western-Hungary. Overview on the present stage of Research in Zala County, Hungary. In: J. Kovárník (ed.): Centenary of Jaroslav Palliardi's Neolithic and Aeneolithic relative chronology (1914-2014). Hradec Králové - Ústí nad Orlicí 2016, 309-336.

Bátora u. a. 2008 - J. Bátora/B. Eitel/F. Falkenstein/ K. Rassmann: Fidvár bei Vráble. Eine befestigte Zentralsiedlung der Frühbronzezeit in der Slowakei. In: J. Czebreszuk/S. Kadrow/J. Müller (eds.): Defensive Structures from Central Europe to the Aegean in the $3^{\text {rd }}$ and $2^{\text {nd }}$ millenia BC. Poznań - Bonn 2008, 97-107.

Bátora u. a. 2012 - J. Bátora/A. Behrens/J. Gresky/ M. Ivanova/K. Rassmann/P. Tóth/K. Winkelmann: The Rise and Decline of the Early Bronze Age Settlement Fidvár near Vráble, Slovakia. In: J. Kneisel/W. Kirleis/ M. Dal Corso/N. Taylor/V. Tiedtke (eds.): Collapse or Continuity? Environment and Development of Bronze Age Human Landscapes. Bonn 2012, 111-129.

Bátora/Tóth/Rassmann 2015 - J. Bátora/P. Tóth/K. Rassmann: Centrálne sídlisko zo staršej doby bronzovej vo Vrábloch. In: J. Bátora/P. Tóth (ed.): Ked’ bronz vystriedal med'. Zborník príspevkov z XXIII. medzinárodného sympózia "Staršia doba bronzová v Čechách, na Morave a na Slovensku“. Levice 8.-11. októbra 2013. Bratislava - Nitra 2015, 123-138.

Dani 2012 - J. Dani: Fortified Tell Settlements from the Middle Bronze Age in the Hungarian Reach of the Berettyó Valley. In: M. Jaeger/J. Czebreszuk/K. P. Fischl (eds.): Enclosed Space - Open Society. Contact and Exchange in the Context of Bronze Age Fortified Settlements in Central Europe. Poznań 2012, 27-37.

Dani u. a. 2019 - J. Dani/S. Puszta/G. Márkus/M. Bálint/ I. Bacskai: Middle Bronze Age Settlement Network in the Polgár Microregion. In: K. P. Fischl/T. L. Kienlin (eds.): Beyond Divides - The Otomani-Füzesabony Phenomenon. Current Approaches to Settlement and Burial in the North-eastern Carpathian Basin and Adjacent Areas. UPA 345. Bonn 2019, 165-194.

David 2010 - W. David: Nascita e crollo della civiltà dei Tell dell'antica età del Bronzo nel bacino carpatico ed indizi di possibili, analoghi fenomeni culturali coevi nella regione dell'alto e medio Danubio nella prima metà del II millenio a.C. In: A. Cardarelli/A. Cazzella/ M. Frangipane/R. Peroni (ed.): Le ragioni del cambiamento. Atti del convegno internazionale „Le ragioni del cambiamento" Roma, 15-17 Giugno 2006. Scienze dell'antichità. Storia, Archeologia, Antropologia 15. Rom 2010, 563-594.

Falkenstein u. a. 2008 - F. Falkenstein/J. Bátora/B. Eitel/ K. Rassmann: Fidvár bei Vráble. Archäologische Prospektionen auf einer befestigten Zentralsiedlung der Frühbronzezeit in der Slowakei. Mitteilungen der Berliner Gesellschaft für Anthropologie 29, 2008, 39-50.

Fischl/Kienlin 2013 - K. P. Fischl/T. L. Kienlin: Results of a Systematic Survey Programme on the Hatvan Sites of Emőd-Nagyhalom and Tard-Tatárdomb in Northern
Hungary. Acta Archaeologica Academiae Scientiarum Hungaricae 64, 2013, 5-32.

Fischl/Olexa 2019 - K. P. Fischl/L. Olexa: The Settlement Network of the Otomani-Füzesabony Cultural Complex in the Southern Hornád Valley and the Košice Basin. In: K. P. Fischl/T. L. Kienlin (eds.): Beyond Divides - The Otomani-Füzesabony Phenomenon. Current Approaches to Settlement and Burial in the North-eastern Carpathian Basin and Adjacent Areas. UPA 345. Bonn 2019, 121-142. Fischl/Kienlin/Seres 2012 - K. P. Fischl/T. L. Kienlin/N. Seres: Bronzezeitliche (RBA1-2) Siedlungsforschungen auf der Borsoder Ebene und im Bükk-Gebirge. Überblick und neue Ergebnisse. A Herman Ottó Múzeum Évkönyve 51, 2012, 23-43

Fischl/Kienlin/Pusztai 2016 - K. P. Fischl/T. L. Kienlin/ T. Pusztai: Bronze Age Settlement Research in the Southern Borsod Region. Hungarian Archaeology. E-Journal, Summer 2016, 2016, 1-8.

Furholt u. a. 2014 - M. Furholt/J. Bátora/I. Cheben/H. Kroll/ K. Rassmann/P. Tóth: Vráble-Vel'ké Lehemby: Eine Siedlungsgruppe der Linearkeramik in der Südwestslowakei. Vorbericht über die Untersuchungen der Jahre 2010 und 2012 und Deutungsansätze. Slovenská archeológia 62, 2014, 227-266.

Furmánek/Marková 1992 - V. Furmánek/K. Marková: Siedlung des Tell-Typs in Včelince. In: J. Herrmann (Hrsg.): Heinrich Schliemann. Grundlagen und Ergebnisse moderner Archäologie 100 Jahre nach Schliemanns Tod. Berlin 1992, 293-303.

Furmánek/Marková 1998 - V. Furmánek/K. Marková: Osobitosti sídliska tellového typu vo Včelinciach. Slovenská archeológia 46, 1998, 205-224.

Furmánek/Marková 2001 - V. Furmánek/Marková: Beitrag der Ausgrabung der Siedlung in Včelince zur Problematik der Bronzezeit im Theißgebiet. In: C. Kacsó (Hrsg.): Der nordkarpatische Raum in der Bronzezeit. Symposium Baia Mare, 7.-10. Oktober 1998. Bibliotheca Marmatia 1. Baia Mare 2001, 105-118.

Furmánek/Marková 2008 - V. Furmánek/K. Marková: Včelince. Archív dávnej minulosti. Nitra 2008.

Füzesi/Raczky 2018 - A. Füzesi/P. Raczky: Öcsöd-Kováshalom. Potscape of a Late Neolithic site in the Tisza region. In: D. Bartus (ed.): Dissertationes Archaeologicae ex Instituto Archaeologico Universitatis de Rolando Eötvös nominatae. Ser. 3. No. 6. Budapest 2018, 43-146.

Gašaj 2002 - D. Gašaj: Osady warowne i życie gospordarcze. In: J. Gancarski (red.): Między Mykenami a Bałtykiem. Kultura Otomani-Füzesabony. Krosno 2002, 21-52.

Gogâltan/Cordoș/Ignat 2014 - F. Gogâltan/C. Cordoș/A. Ignat: Bronze Age tell, tell-like and mound-like settlements on the eastern frontier of the Carpathian Basin. History of research. Cluj-Napoca 2014.

Görsdorf/Marková/Furmánek 2004-J. Görsdorf/K. Marková/ V. Furmánek: Some new $14 \mathrm{C}$ data to the Bronze Age in the Slovakia. Geochronometria 23, 2004, 79-91.

Gyucha u. a. 2015-A. Gyucha/R. W. Yerkes/W. A. Parkinson/ A. Sarris/N. Papadopoulos/R. P. Duffy/B. R. Salisbury: Settlement Nucleation in the Neolithic. A Preliminary Report of the Körös Regional Archaeological Project's Investigations at Szeghalom-Kovácshalom and Vésztő-Mágor. In: S. Hansen/P. Raczky/A. Anders/ A. Reingruber (eds.): Neolithic and Copper Age between the Carpathians and the Aegean Sea. Chronologies and 
technologies from the $6^{\text {th }}$ to the $4^{\text {th }}$ millennium BCE. Archäologie in Eurasien 31. Bonn 2015, 129-142.

Jaeger u. a. 2018 - M. Jaeger/G. Kulcsár/N. Tylor/R. Staniuk: Kakucs-Turján. A Middle Bronze Age multi-layered fortified settlement in Central Hungary. Bonn 2018.

Kalicz 1968 - N. Kalicz: Die Frühbronzezeit in Nordost-Ungarn. Budapest 1968.

Kalicz 1985 - N. Kalicz: Über das spätneolithische Siedlungswesen in Ungarn. Béri Balogh Ádám Múzeum Évkönyve 13, 1985, 127-138.

Kalicz/Raczky 1990 - N. Kalicz/P. Raczky: Das Spätneolithikum im Theissgebiet. Eine Übersicht zum heutigen Forschungsstand aufgrund der neuesten Ausgrabungen. In: W. Meier-Arendt (Hrsg.): Alltag und Religion. Jungsteinzeit in Ostungarn. Frankfurt am Main 1990, 11-30.

Kienlin 2018 - T. L. Kienlin: I. Borsod Region Bronze Age Settlement: 'Diversity in Uniformity'. In: T. L. Kienlin/ K. P. Fischl/T. Pusztai: Borsod Region Bronze Age Settlement (BORBAS). Catalogue of the Early to Middle Bronze Age Tell Sites Covered by Magnetometry and Surface Survey. Bonn 2018, 11-98.

Kienlin/Fischl/Marta 2017 -T. L. Kienlin/K. P. Fischl/L. Marta: Exploring Divergent Trajectories in Bronze Age Landscapes. Tell Settlement in the Hungarian Borsod Plain and the Romanian Ier Valley. Ziridava 31, 2017, 93-128.

Kienlin/Fischl/Pusztai 2018 - T. L. Kienlin/K. P. Fischl/ T. Pusztai: Borsod Region Bronze Age Settlement (BORBAS). Catalogue of the Early to Middle Bronze Age Tell Sites Covered by Magnetometry and Surface Survey. Bonn 2018.

Kienlin/Lie/Fischl 2019 - T. L. Kienlin/M. A. Lie/K. P. Fischl: Emőd-Nagyhalom. A Non-invasive Approach to the Multi-phase Enclosure and Outer Settlement of a Bronze Age Tell Site in North-eastern Hungary. In: K. P. Fischl/T. L. Kienlin (eds.): Beyond Divides - The Otomani-Füzesabony Phenomenon. Current Approaches to Settlement and Burial in the North-eastern Carpathian Basin and Adjacent Areas. UPA 345. Bonn 2019, 195-229.

Kovárník 2015 - J. Kovárník: Opevňovací příkop ze starší doby bronzové u Šatova, okr. Znojmo. Další případy obloukovitých příkopů zjištěných leteckým výzkumem. In: J. Bátora/P. Tóth (ed.): Ked' bronz vystriedal med'. Zborník príspevkov z XXIII. medzinárodného sympózia "Staršia doba bronzová v Čechách, na Morave a na Slovensku“. Levice 8.-11. októbra 2013. Bratislava - Nitra 2015, 105-122.

Mesterházy u. a. 2019 - G. Mesterházy/G. Serlegi/ B. Vágvölgyi/A. Füzesi/P. Raczky: A szociális folyamatok színterei Polgár-Csőszhalom késő neolitikus településének összefüggéseiben. Archaeologiai Értesítő 144, 2019, 1-32.

Olexa 1982 - L. Olexa: Siedlungen aus der Bronzezeit in Nižná Myšla in der Ostslowakei. In: B. Chropovský/ J. Herrmann (Hrsg.): Beiträge zum bronzezeitlichen Burgenbau in Mitteleuropa. Berlin - Nitra 1982, 331-334.

Olexa 2003 - L. Olexa: Nižná Myšla. Osada a pohrebisko z doby bronzovej. Košice 2003.

Parkinson u. a. 2004 - W. A. Parkinson/A. Gyucha/R. W. Yerkes/A. Sarris/M. Hardy/M. Morris: Settlement Reorganization at the End of the Neolithic in Central Europe. Recent Research in the Körös River Valley, Southeastern Hungary. Eurasian Prehistory 2, 2004, 57-73.

Pásztor/Barna/Zotti 2015 - E. Pásztor/J. P. Barna/G. Zotti: Neolithic Circular Ditch Systems ('Rondels') in Central
Europe. In: C. L. N. Ruggles (ed.): Handbook of Archaeoastronomy and Ethnoastronomy. New York 2015, 1317-1326.

Raczky 1987 - P. Raczky: The Late Neolithic of the Tisza Region. Budapest - Szolnok 1987.

Raczky 2019 - P. Raczky: Cross-Scale Settlement Morphologies and Social Formations in the Neolithic of the Great Hungarian Plain. In: A. Gyucha (ed.): Coming Together. Comparative Approaches to Population Aggregation and Early Urbanization. Albany 2019, 259-293.

Raczky/Anders 2006 - P. Raczky/A. Anders: Social dimensions of the Late Neolithic settlement of Polgár-Csőszhalom (eastern Hungary). Acta Archaeologica Academiae Scientiarum Hungaricae 57, 2006, 17-33.

Raczky/Anders 2008 - P. Raczky/A. Anders: Late Neolithic spatial differentiation at Polgár-Csőszhalom. In: D. Bailey/A. Whittle/D. Hofmann (eds.): Living Well Together. Settlement and materiality in the Neolithic of southeast and central Europe. Oxford 2008, 122-125.

Raczky/Anders 2009 - P. Raczky/A. Anders: Régészeti kutatások egy késő neolitikus településen. Polgár-Bosnyákdomb. Előzetes jelentés. Archaeologiai Értesító 134, 2009, 5-21.

Raczky/Anders 2010a - P. Raczky/A. Anders: The times they are a-changin'. Revisiting the chronological framework of the Late Neolithic settlement complex at Polgár-Csőszhalom. In: J. Šuteková/P. Pavúk/P. Kalábková/ B. Kovár (eds.): PANTA RHEI. Studies on the Chronology and Cultural Development of South-Eastern and Central Europe in Earlier Prehistory. Presented to Juraj Pavúk on Occasion of his $75^{\text {th }}$ Birthday. Bratislava 2010, 357-378.

Raczky/Anders 2010b - P. Raczky/A. Anders: Activity loci and data for spatial division at a Late Neolithic site-complex (Polgár-Csőszhalom: a case study). In: S. Hansen (Hrsg.): Leben auf dem Tell als soziale Praxis. Beiträge des Internationalen Symposiums in Berlin vom 26.-27. Februar 2007. Kolloquien zur Vor- und Frühgeschichte 14. Bonn 2010, 143-163.

Raczky/Anders 2012 - P. Raczky/A. Anders: Neolithic enclosures in Eastern Hungary and their survival into the Copper Age. In: F. Bertemes/H. Meller (Hrsg.): Neolithische Kreisgrabenanlagen in Europa. Tagungen des Landesmuseums für Vorgeschichte Halle 8. Halle (Saale) 2012, 271-309.

Raczky/Anders 2014 - P. Raczky/A. Anders: Szentpéterszeg-Kovadomb. Egy késő neolitikus lelőhely tér-képei. In: A. Anders/C. Balogh/A. Türk (szerk.): Avarok pusztái. Régészeti tanulmányok Lörinczy Gábor 60. születésnapjára. Budapest 2014, 23-42.

Raczky/Füzesi 2016 - P. Raczky/A. Füzesi: Öcsöd-Kováshalom. A retrospective look at the interpretations of a Late Neolithic site. In: D. Bartus (ed.): Dissertationes Archaeologicae ex Instituto Archaeologico Universitatis de Rolando Eötvös nominatae. Ser. 3. No. 4. Budapest 2016, 9-42.

Raczky/Sebők 2014 - P. Raczky/K. Sebők: The outset of Polgár-Csőszhalom tell and the archaeological context of a special central building. In: S. Forțiu/A. Cîntar (ed.): Arheovest II. In honorem Gheorghe Lazarovici. Interdisciplinaritate in Arheologie. Timişoara, 6 decembrie 2014. Szeged 2014, 51-100.

Raczky/Fodor/Mester 2010 - P. Raczky/I. Fodor/Z. Mester: Régészeti kutatások Hajdúböszörmény-Pródi-halmon. Archaeologiai Értesítő 135, 2010, 161-182.

Raczky/Anders/Bartosiewicz 2011 -P. Raczky/A. Anders/L. Bartosiewicz: The enclosure system of Polgár-Csőszhalom 
and its interpretation. In: S. Hansen/J. Müller (Hrsg.): Sozialarchäologische Perspektiven. Gesellschaftlicher Wandel 5000-1500 v. Chr. zwischen Atlantik und Kaukasus. Internationale Tagung 15.-18. Oktober 2007 in Kiel. Archäologie in Eurasien 24. Darmstadt 2011, 57-79.

Raczky/Anders/Siklósi 2014 - P. Raczky/A. Anders/Z. Siklósi: Trajectories of continuity and change between the Late Neolithic and the Copper Age in Eastern Hungary. In: W. Schier/F. Draşovean (eds.): The Neolithic and Eneolithic in Southeast Europe. New Approaches to dating and cultural dynamics in the $6^{\text {th }}$ to $4^{\text {th }}$ millennium BC. PAS 28. Rahden/ Westf. 2014, 319-346.

Raczky u. a. 2015 - P. Raczky/A. Anders/K. Sebők/ P. Csippán/Z. Tóth: The times of Polgár-Csőszhalom. Chronologies of human activities in a Late Neolithic settlement in northeastern Hungary. In: S. Hansen/ P. Raczky/A. Anders/A. Reingruber (eds.): Neolithic and Copper Age between the Carpathians and the Aegean Sea. Chronologies and technologies from the $6^{\text {th }}$ to the $4^{\text {th }}$ millennium BCE. Archäologie in Eurasien 31. Bonn 2015, 21-48.

Raczky/Füzesi/Anders 2018 - P. Raczky/A. Füzesi/A. Anders: Domestic and Symbolic Activities on a Tell-Like Settlement at Öcsöd-Kováshalom in the Tisza Region. In: K. A. Romstorfer (ed.): Volume of the International Symposium. The Image of Divinity in the Neolithic and Eneolithic. Ways of Communication. Suceava 2018, 117-140.

Rassmann u. a. 2017 - K. Rassmann/S. Reiter/J. Bátora/ N. Müller-Scheeßel: The Vráble Toolbox. A Multidisciplinary Investigation of Settlement Change. In: B. Heeb/A. Szentmiklosi/R. Krause/M. Wemhoff (eds.): Fortifications. The Rise and Fall of Defended Sites in Late Bronze Age and Early Iron Age of South-East Europe. Berliner Beiträge zur Vor- und Frühgeschichte 21. Berlin 2017, 79-90.

Rassmann u. a. 2018 - K. Rassmann/J. Bátora/N. Müller-Scheeßel/S. Reiter/M. Ivanova/A. Behrens/K. Radloff/

Manuskript angenommen am 24. 7. 2020

Abstract translated by Tobias L. Kienlin

Súhrn preložila Anita Kozubová

Dr. Klára P. Fischl

Történettudományi Intézet

Miskolci Egyetem

Miskolc-Egyetemváros B/2

$\mathrm{H}-3515$ Miskolc

fklari@gmail.com

Gian-Luca Paul, B.A.

Institut für Ur- und Frühgeschichte

Universität zu Köln

Weyertal 125

D - 50931 Köln

gian-luca.paul@gmx.de
M. Bača: Tracing Taphonomic Processes. Multiple Layer Analysis of Ceramic Distribution from Surface Collection and Excavation at the Early Bronze Age Settlement of Vráble-Fidvár. Slovenská archeológia 66, 2018, 219-234.

Schlütz/Bittmann 2016 - F. Schlütz/F. Bittmann: Dating Archaeological Cultures by Their Moats? A Case Study from the Early Bronze Age Settlement Fidvár near Vráble, SW Slovakia. Radiocarbon 58, 2016, 331-343.

Skorna/Kalmbach/Bátora 2018 - H. Skorna/J. Kalmbach/ J. Bátora: Vráble, Slowakei. Herausbildung und Niedergang des frühbronzezeitlichen Siedlungszentrums. Untersuchungen zu Wirtschaft, Sozialstruktur und politischer Organisation eines Sozialverbandes und seines Umfeldes. Überblick und die Arbeiten in der Siedlung 2017. DAI e-Forschungsbericht 2018, 101-108.

Staniuk u. a. 2020 - R. Staniuk/M. Jaeger/G. Kulcsár/ N. Taylor/J. Niebieszczański/J. Müller: Moving bottom-up: The case study of Kakucs-Turján and its implications for studies of multi-layered, Bronze Age settlements in the Carpathian Basin. In: A. Blanco-González/T. L. Kienlin (eds.): Current approaches to tells in the prehistoric Old World. Oxford 2020, 57-72.

Szeverényi/Kulcsár 2012 - V. Szeverényi/G. Kulcsár: Middle Bronze Age Settlement and Society in Central Hungary. In: M. Jaeger/J. Czebreszuk/K. P. Fischl (eds.): Enclosed Space - Open Society. Contact and Exchange in the Context of Bronze Age Fortified Settlements in Central Europe. Poznań 2012, 287-351.

Točík 1968 - A. Točík: K otázce interpretace pravěkých staveb. Archeologické rozhledy 20, 1968, 490-510.

Vavák u. a. 2015 - J. Vavák/P. Jelínek/J. Hlavatá/ L. Illášová: Doklady metalurgie na opevnenom sídlisku mad’arovskej kultúry v Budmericiach. In: J. Bátora/ P. Tóth (ed.): Ked'bronz vystriedal med'. Zborník príspevkov z XXIII. medzinárodného sympózia „Staršia doba bronzová v Čechách, na Morave a na Slovensku“. Levice 8.-11. októbra 2013. Bratislava - Nitra 2015, 157-186.
Prof. Dr. Tobias L. Kienlin

Institut für Ur- und Frühgeschichte

Universität zu Köln

Weyertal 125

D - 50931 Köln

tkienlin@uni-koeln.de 


\title{
Vráble-Fidvár v kontexte: predchodcovia a paralely demarkácie
}

\author{
Klára P. Fischl- Gian-Luca Paul- Tobias L. Kienlin
}

\section{SÚHRN}

Príspevok obsahuje krátky prehlad a porovnanie demarkácií tellových a tellovitých sídlisk rôznych skupín staršej doby bronzovej v Karpatskej kotline a ich vonkajšieho osídlenia. Najmä z pohladu územia dnešného Mad’arska a s akcentom na posledné výsledky projektu BORBAS („,Osídlenie boršodského regiónu v dobe bronzovej“) sa autori príspevku venujú štúdiu štrukturálnych podobnostía variability spôso- bu, akým spoločenstvá staršej doby bronzovej v sledovanom priestore uzatvárali svoje sídliská alebo ich časti a ako sa tieto sídliská vyvíjali v čase. Osobitná pozornost̉ je v príspevku venovaná demarkáciám viacvrstvových neolitických sídlisk ako vzdialených predchodcov fenoménu demarkácií z doby bronzovej, ktoré okrem štrukturálnej podobnosti podliehali podobným zmenám v čase a úpravách. 